test könne dann sicher zur Diagnose Allergie oder nicht allergische Intoleranzreaktion führen, bei negativem Provokationstest sind weitere Differenzialdiagnosen zu verfolgen. So ließe sich zum Beispiel bei einem Patienten, der auf die Behandlung einer Wundinfektion mit Clindamycin ein Exanthem entwickelt, eine Allergie gegen das Mittel durch einen positiv ausfallenden Provokationstest nach negativem Befund im Hauttest nachweisen. Wird ein Patient bei einem Atemwegsinfekt mit Amoxicillin behandelt und entwickelt daraufhin ein Exanthem, könne bei negativen Befunden in Haut- und Provokationstests die Differenzialdiagnose Virusexanthem gestellt werden.

Brehlers Argumentation hingegen zielte auf den Begriff des "Goldstandards". Hierbei handelt es sich laut seinen Ausführungen per definitionem um "das diagnostische Verfahren, das im gegebenen Fall die bewährteste und beste Lösung darstellt"
- ein nach aktueller Evidenzlage allgemeingültiger und maßgebender Standard, nach dem sich alle zu richten haben. Davon könne im Falle des Provokationstests keine Rede sein. So kämen die meisten Patienten wegen bereits lange zurückliegender Arzneimittelreaktionen und könnten oft weder den Grund für die damals erfolgte Behandlung noch die angewendeten Mittel benennen, die Hautveränderungen beschreiben oder Angaben zum zeitlichen Verlauf und der Behandlung machen. Eine Abklärung von Arzneimittelreaktionen mit Provokationstest müsse jedoch innerhalb eines Jahres erfolgen. Zudem könne dem Anspruch des Patienten, mittels Provokationstest zu erkennen, welche Medikamente künftig vertragen würden, nicht Rechnung getragen werden - keine prospektiven, randomisierten, kontrollierten Studien hätten gezeigt, dass die in der Provokation vertragenen Medikamente auch später noch gut vertragen werden.
Sich auf den Befund des Provokationstests zu verlassen, wiege in falscher Sicherheit: Oft benötigten die Arzneimittelreaktionen Kofaktoren wie Infekt, Anstrengung oder eine bestimmte Komedikation. SpättypReaktionen werden oft nicht durch die Medikamentengabe an einem Tag ausgelöst, eine Provokation über mehrere Tage hinweg jedoch sei unverantwortlich, weil sie eine Sensibilisierung induzieren könne. Als Alternative definierte Brehler einen neuen Goldstandard für die Diagnose von Arzneimittelallergien: Die vollständige Anamnese und Dokumentation auftretender Reaktionen, Hauttests und gegebenenfalls In-vitro-Tests in einem geeigneten Zeitraum. Erst danach könne über die Indikation zum Provokationstest nachgedacht werden.

Kathrin von Kieseritzky

AllergoArena: „Arzneimittelallergie 2016:

Provokationstest ist Goldstandard“

\title{
Betalaktamantibiotika und Schmerzmittel
}

— Wie Prof. Dr. Axel Trautmann, Würzburg, ausführte, können Amoxicillinallergien auf unterschiedliche Weise in Erscheinung treten: Als IgE-vermittelte Sofortreaktionen mit Urtikaria und Angioödemen bis hin zu höhergradigen anaphylaktischen Reaktionen, die innerhalb weniger Minuten auftreten und sich in wenigen Stunden zurückbilden, ebenso wie als T-Lymphozyten-vermittelte Spätreaktionen mit unkomplizierten Exanthemen, verschiedenen morphologischen Exanthemvarianten, exanthematischen Systemreaktionen und bullösen Hautreaktionen, die sich erst nach mehreren Stunden bis Tagen bilden und mehrere Tage bis Wochen anhalten können.

Treten nach der Einnahme eines Betalaktamantibiotikums Symptome wie Urtikaria und/ oder Exanthem auf, und erweisen sich die Haut- (Prick, intrakutan, epikutan) und IgETests als positiv, ist keine weitere Diagnostik in Form einer Provokation notwendig. Sind dagegen die Haut- und IgE-Tests negativ, kann eine kontrollierte Provokationstestung erfolgen. In bis zu $95 \%$ der Fälle falle dann auch der Provokationstest negativ aus, so Trautmann. Doch 100\%ige Sicherheit biete nur die kontrollierte Provokation. Bei Hinweisen auf schwere Reaktionen sollte auf eine ambulante Provokation verzichtet werden, die Patienten sind am besten in ein entsprechend spezialisiertes Zentrum zu überweisen. Erweisen sich die Provokationstests als positiv, ist die Allergie damit eindeutig nachgewiesen, sind sie negativ, kann auch die Allergie klar ausgeschlossen werden.

Eine Allergiediagnostik kann das auslösende Penicillin oder Cephalosporin mit Sicherheit identifizieren. Das Allergen bilden in der Regel die Aminobenzyl-Seitenketten, die bei den Cefalosporinen der neueren Generationen verändert worden sind, weshalb diese Mittel oft vertragen werden. Trautmann verwies zudem darauf, dass auch der Ausschluss einer Penicillinallergie bedeutsam sei, weil dies im weiteren Lebensverlauf notwendig werdende Antibiotikatherapien erleichtert.

Auch Schmerzmittel, die nach Aussage von Prof. Dr. Bettina Wedi, Hannover, weltweit am häufigsten eingesetzten Pharmaka überhaupt, führen Überempfindlichkeitsreaktionen herbei. Dabei kann jedes Schmerzmittel jeden Reaktionstyp auslösen. Mit Abstand am häufigsten treten bei NSAID-Überempfindlichkeit Urtikaria und Angioödem auf, doch auch anaphylaktische
Reaktionen sind mit $25 \%$ nicht selten. Um die Diagnose zu sichern, die auslösenden Mittel zu identifizieren, Kreuzreaktionen auszuschließen und Alternativen für die Therapie mit Schmerzmitteln herauszufiltern, sind kontrollierte Provokationstests laut Wedi unerlässlich Kathrin von Kieseritzky

Curriculum der Allergologie: „Moderne Diagnostik von ..."

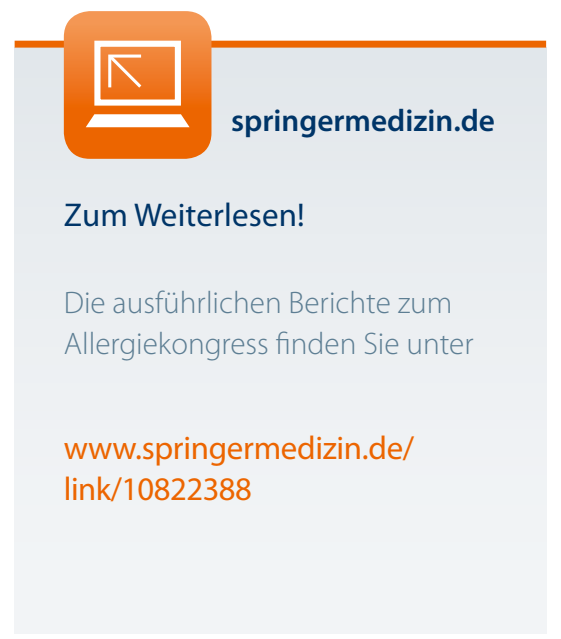

